\title{
PROSPECTIVA TRANSDISCIPLINARIA Y ORGANIZACIÓN DEL CONOCIMIENTO: UNA APROXIMACIÓN EPISTÉMICA A LA EDUCACIÓN SUPERIOR EN LA REGIÓN DE MAGALLANES, CHILE
}

IVÁN G. OLIVA-FIGUEROA ${ }^{a}$, WALTER M. MOLINA-CHÁVEZ JOHN J. QUINTERO-TAPIAc \& NICOLÁS S. DÍAZ-BARRERA

\section{RESUMEN ${ }^{1}$}

El artículo expone los principales lineamientos teóricos asociados a un programa de investigación científica en torno a las posibilidades y condicionantes para el desarrollo de dinámicas inter-transdisciplinares en la Educación Superior en Chile. Desde esta base, la investigación de campo tuvo por objetivo interpretar y cartografiar las amplias regiones de sentido en torno a la noción de transdisciplinariedad desplegadas en diversos campos disciplinarios en la Educación Superior en la Región de Magallanes. Se consideraron estrategias metodológicas complementarias para la generación y análisis de información. Los resultados plantean que la noción de inter-transdisciplina es un elemento de sentido con amplio valor social y académico para los informantes claves participantes de la investigación. No obstante, esta amplia y distribuida valoración, se expresa de formas muy diversas en términos de los elementos de sentidos adyacentes a ella, en correlato con los diversos campos disciplinares y el rol (académicos y estudiantes) que desempeñan los informantes claves.

PALABRAS CLAVE: interdisciplina, transdisciplina, universidad, complejidad.

\section{TRANSDISCIPLINARY PROSPECTIVE AND KNOWLEDGE ORGANIZATION: AN EPISTEMYC APPROACH TO HIGHER EDUCATION IN THE MAGALLANES REGION, CHILE}

\section{ABSTRACT}

This paper presents the main theoretical guidelines associated with a program of scientific research on the possibilities and constraints for the development of inter-transdisciplinary dynamics in Higher

1 El artículo forma parte del programa de investigación asociado al proyecto Fondecyt-Regular 1150923 "Interfaces universidad $\leftarrow \rightarrow$ sociedad desde la prospectiva transdisciplinaria: Metodología de redes complejas y minería de datos aplicadas a dinámicas de organización del conocimiento a escala social".

a Universidad Austral de Chile. Campus Isla Teja S/N. Valdivia, Chile. $\ \backslash$ ivanoliva@uach.cl

b Universidad de Magallanes. Facultad de Educación y Ciencias Sociales. Punta Arenas, Chile. walter.molina@umag.cl

c Universidad del Cauca. Facultad de Ciencias Naturales, Exactas y de la Educación. Popayán, Colombia. jjquintero@unicauca. edu.co

d Centro de Investigación en Educación (CIE) Universidad Bernardo O'Higgins. ndiaz.lenguaje@gmail.com 
Education in Chile. On this basis, the field research aim was to interpret and map the extensive regions of sense around the notion of transdisciplinarity deployed in various disciplinary fields in Higher Education in the Magallanes Region. Complementary methodological strategies were considered for the generation and analysis of information. The results suggest that the notion of inter-disciplinarity is an element of sense/direction with broad social and academic value for the informants, key participants of this research. However, this wide and distributed evaluation, is expressed in a variety of ways in terms of the elements of senses adjacent to it, in correlation with the various disciplinary fields and the role (scholars and students) that key informants play.

KEY WORDS: interdiscipline, transdiscipline, university, complexity.

\section{ANTECEDENTES GENERALES}

Las formas de interacción y dinámicas de organización del conocimiento en los procesos de formación, investigación e interacción con la sociedad de las universidades contemporáneas configuran una problemática comunicacional, epistemológica, curricular y política con profundas implicancias para el desarrollo social de los países en términos de sus fenómenos de segregación y desigualdad. En este contexto, la noción de organización del conocimiento asume una dimensión reticular y compleja, dado que significa asumir una perspectiva de investigación orientada a comprender, analizar y modelar una amplia gama de patrones cognitivos de significación locales $e$ interacciones sociales emergentes. En este ámbito, la reflexión en torno a la pertinencia epistemológica de los procesos formativos e investigativos de la educación terciaria en el país, no sólo es relevante para establecer métricas o indicadores cuantitativos, sino también para el modelado y cartografiado de una diversidad cualitativa de patrones de organización del conocimiento en diversos campos disciplinarios.

El énfasis en el entendimiento de las dinámicas de organización del conocimiento en diversos campos disciplinarios, abre un marco teórico que integra elementos de la Teoría General de los Sistemas (von Bertalanffy, 1976; Capra, 1998, 2002), Cibernética de Segundo Orden (von Foerster, 1996), epistemología de la complejidad (Morin, 1992, 1995, 2006) y transdisciplinaridad (Somerville \& Rapport, 2000; Motta, 2002; Nicolescu, 2008; Lenoir, 2013). Asimismo, parte del desarrollo teórico, como la discusión específica de las tipologías de los recursos conceptuales empleados y la proyección metodológica de nuestro programa de investigación, ha sido tratado en trabajos anteriores (Oliva, 2008; Díaz et al. 2013; Oliva et al. 2014; Koch et al. 2015).
Desde este marco de referencia, los patrones de generación y comunicación del conocimiento, en sus diversas dimensiones, han permeado progresivamente los modelos sociológicos y económicos a escala global, a la vez de impactar en diversas aristas de la teoría organizacional, con especial énfasis en aquellas denominadas knowledge-intensive organizations (Cross \& Parker, 2004). Entre éstas, podemos situar a universidades complejas que, producto de sus objetivos institucionales, desarrollan actividades de docencia, investigación y extensión.

Es evidente que el carácter complejo adscrito a este tipo de organizaciones, no sólo puede estar definido en torno al desarrollo paralelo de estas tres áreas, sino que a la vez debe considerar sus efectos de interacción, coordinación y emergencia de procesos complejos de generación y comunicación de conocimiento. En este escenario es posible dar cuenta de la emergencia de las prospectivas inter y transdisciplinaria (Piaget, 1970; Morin, 1998, 2006, Nicolescu, 1996, 2008; Motta, 2000, 2002; Max-Neef, 2003; Greckhamer et al. 2008), en educación superior, a modo de abordar las profundas fracturas epistemológicas y comunicacionales entre los diversos sistemas de conocimiento y sus formas de organización. Pese a su polisemia, las nociones de inter y transdisciplina abordan transversalmente las dinámicas de quiebre y articulación entre los diversos sistemas de conocimiento, dando forma de este modo, a una perspectiva epistemológica crítica en torno a la hegemonía de patrones de hiper-especialización en la organización del conocimiento científico. Asimismo, es posible postular que las organizaciones con mayor grado de densidad relacional en sus procesos de comunicación inter y transdisciplinarios, amplían sus posibilidades y rangos cualitativos de 
interacción y vinculación efectiva con la sociedad en la cual se inscriben, dando forma a interfaces complejas y recursivas con su entorno.

Desde este planteamiento, sostenemos que existen importantes grados de fractura cognitiva en las redes que sustentan los procesos de generación, comunicación y organización del conocimiento en la interfaz universidad $\leftrightarrow \rightarrow$ sociedad, limitando de este modo el surgimiento de dimensiones de aprendizaje social y generación de conocimiento con mayores grados de complejidad cualitativa, flexibilidad y transdisciplinariedad. Este nodo crítico se expresa, según Flores-Malagón et al. (2002), en que los programas disciplinares al interior de las universidades pierdan terreno ante los nuevos programas transdisciplinares e interdisciplinares $y$, con ello, el problema de las disciplinas como referentes adecuados para el trabajo intelectual se configure como uno de los puntos de ruptura práxica más importantes del siglo XXI. Añaden además que los estudiantes de distintos contextos formativos se distancian de currículos cerrados y especializados, a la vez que el mercado laboral envía señales ambiguas y genera una demanda que no se reduce a especialistas disciplinares, requiriendo cada vez más profesionales con una formación integral, aún más fuerte que la disciplinar.

En este contexto, los objetivos del programa de investigación han estado orientados, en términos globales, al análisis de procesos de disyunción y segregación de capacidades cognitivas a escala organizacional en el ámbito de la educación superior en Chile, a través de enfoques metodológicos complementarios de naturaleza cualitativa $y$ cuantitativa. De este modo, el énfasis ha estado orientado a la detección y comprensión de nodos críticos en los efectos de interactividad intertransdisciplinaria en diversos contextos universitarios regionales, atendiendo a sus procesos de generación y comunicación del conocimiento.

Desde la base de este programa global, la especificidad de la investigación estuvo orientada a interpretary cartografiar las amplias regiones de sentido en torno a la noción de inter-transdisciplinariedad desplegadas en los diversos campos disciplinarios desarrollados en la Universidad de Magallanes, Chile. En este contexto, fueron relevados los principales sustratos cualitativos en el entendimiento de las posibilidades y condicionantes de la prospectiva inter-transdisciplinaria en los procesos formativos e investigativos de la región.

Dinámicas de interacción interdisciplinar, flexibilidad curricular y movilidad lateral en Educación Superior en Chile

A partir de nuestros proyectos anteriores (Oliva, 2008; Oliva et al. 2014) hemos sostenido que la educación terciaria ha generado profundas disyunciones epistémicas entre los diversos campos disciplinares/profesionales, como correlato de una flexibilidad curricular limitada, sobre-especialización del currículum y sistemas de conocimiento disciplinares autorreferenciados que configuran su legitimidad exclusivamente dentro de sus propios límites fenomenológicos. Asimismo, hemos precisado que una importante deficiencia estructural de los modelos curriculares y la política de educación terciaria, está asociada a la capacidad de las universidades para organizar y comunicar campos disciplinares aislados en un orden más interactivo y complejo, limitando con ello el desarrollo cultural, la lectura crítica de la sociedad y una comprensión integrada de fenómenos intertransdisciplinarios y planetarios.

Lo anterior supone un tránsito hacia un currículo más flexible, sistémico y reflexivo, proyectado desde la base de nuevos territorios temáticos con la incorporación de enfoques multirreferenciales, del diálogo de saberes y metodologías, así como de la articulación de las ciencias, las humanidades y las artes, posibilitando la movilidad vertical y horizontal de estudiantes y profesores (González, 2011). Dentro del marco de especificidad de la Educación Superior en Chile y atendiendo a estándares internacionales, el informe OCDE (2009) ha señalado la flexibilidad limitada y la sobreespecialización del currículum como deficiencias estructurales genéricas del sistema. En relación a la flexibilidad limitada, se señala desde la revisión curricular de una variedad de programas académicos e instituciones, el énfasis de un currículo rígido, con opciones muy limitadas o sin electivos, una vez que el estudiante ha escogido un área de especialización. Esta flexibilidad limitada es uno de los principales impedimentos para la articulación y movilidad entre programas y niveles académicos en Chile. El informe señala también que, salvo pocas excepciones, no se permite la movilidad lateral entre 
programas académicos, incluso dentro de la misma institución. Este factor, combinado con la limitada orientación vocacional en la enseñanza secundaria, tendría gran influencia en las tasas de deserción en la educación terciaria. Complementariamente a este nivel de análisis, se ha señalado que esta flexibilidad restringida se constituye en uno de los principales impedimentos para la articulación y movilidad entre programas y niveles académicos en Chile, en relación a la convergencia curricular entre los niveles técnico superior, profesional y universitario (Gaete \& Morales, 2011).

Desde esta base y la convergencia multidimensional de diversos e imbricados niveles de interpretación (epistemológicos, económicos y curriculares) del sistema, emerge la necesidad y pertinencia de introducir grados de mayor flexibilidad curricular, lo que simultáneamente requiere de la generación y proyección de esquemas de formación inter-transdisciplinares en el sistema de Educación Superior en Chile.

Nuestras investigaciones previas (Oliva et al. 2014), dentro de otras áreas de profundización, diagnosticaron la flexibilidad operativa del currículum optativo, entendida como una de las condicionantes iniciales para la emergencia y configuración de esquemas curriculares más flexibles e intertransdisciplinarios en los procesos formativos de universidades chilenas. Los resultados, dentro de los límites de la investigación, evidenciaron procesos restringidos de desplazamiento e interactividad estudiantil y docente entre los diversos dominios disciplinares (considerando programas de pregrado y postgrado), prevaleciendo transversalmente dinámicas de auto-referencia y aislamiento disciplinar/profesional en términos formativos. Esto puede deberse a que los estudiantes significan en la oferta optativa y flexible, un recurso de profundización y de mayor especialización, antes que la posibilidad de transitar y complementar con otras perspectivas disciplinares su formación profesional particular, inscribiendo de este modo, su especificidad disciplinar en un contexto formativo más global. En sintesis, fue posible postular una significativa rigidez y clausura operativa del currículum flexible, configurando un nodo crítico que condiciona a este nivel, la emergencia de esquemas de docencia con mayores grados de flexibilidad y comunicación inter-

2 Se analizaron más de 240.000 operaciones de inscripción de cursos en tres universidades chilenas, en un periodo transdisciplinaridad en los contextos investigados.

En el contexto de este artículo, creemos necesario triangular estos resultados preliminares (basados fundamentalmente en estadística descriptiva aplicada a bases de registro curricular) con enfoques metodológicos complementarios de orden cualitativo ${ }^{2}$. En otras palabras, dada la naturaleza epistemológica de la prospectiva intertransdiciplinaria para el sistema universitario, no es posible renunciar a la comprensión de los rangos amplios de sentido y las bases paradigmáticas que posibilitan, y a la vez restringen, la emergencia de sistemas de pensamiento más complejos e interactivos en la cultura científica y en el desarrollo multidimensional de la región y el país.

En consecuencia, atendiendo al foco interpretativo y situado del paradigma cualitativo de investigación, se optó por un estudio de casos para efectos del presente reporte de investigación, considerando la especificidad de la educación superior en la Región de Magallanes en Chile y sus dimensiones contextuales asociadas.

\section{Educación Superior en la \\ Región de Magallanes y Antártica Chilena}

La localización geográfica de la Universidad de Magallanes en la Región de Magallanes y Antártica Chilena le otorga particularidades tanto territoriales como humanas a su quehacer institucional, lo cual se refleja en las actividades de docencia de pregrado y postgrado e investigación que desarrolla su cuerpo docente. A modo de ejemplo, es posible mencionar los estudios que se desarrollan en el Parque Etnobotánico Omora (Reserva de la Biósfera Cabo de Hornos) y en el Instituto de la Patagonia en Punta Arenas, donde destacan los estudios arqueológicos en poblamiento humano temprano en la región de Fuego-Patagonia (11.000 años a.n.e) y la configuración contemporánea de las identidades sociales locales en el territorio regional.

Desde una perspectiva local-global, el sistema de educación superior en Magallanes tiene una matrícula de 7.845 estudiantes, distribuidos en instituciones públicas y privadas, que incluyen Universidades, Institutos Profesionales (IP) y Centros de Formación Técnica (CFT). La principal casa de estudios superiores

de tres años, considerando la oferta curricular optativa o flexible de pregrado y postgrado. 


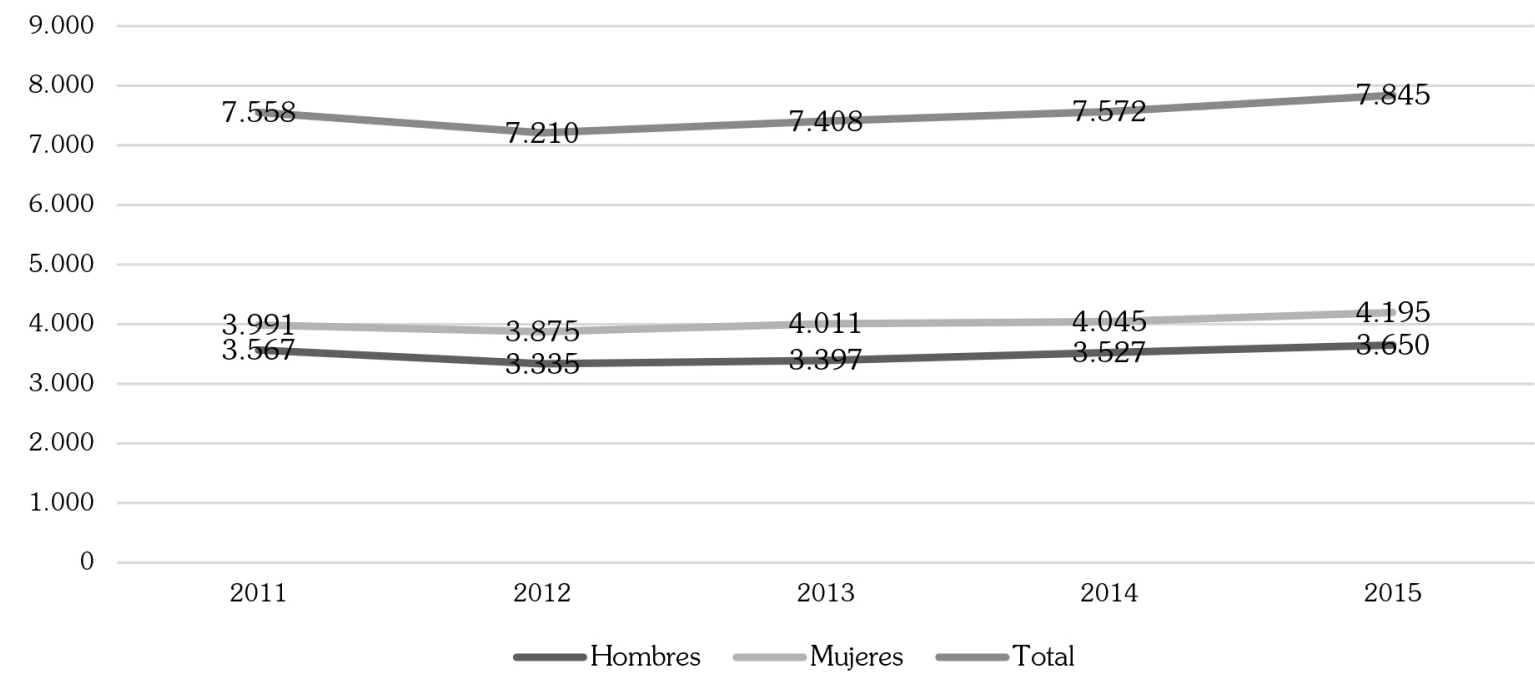

Fig. 1. Evolución de la Matrícula de pregrado región de Magallanes.

Fuente: Estadísticas de la Educación (Ministerio de Educación de Chile, 2015, p. 108).

a nivel regional es la Universidad de Magallanes que concentra una matrícula de pregrado que asciende a 4.091 estudiantes, es decir, un $52.14 \%$ de quienes cursan estudios superiores en la región de Magallanes. Actualmente la Universidad de Magallanes ofrece 31 carreras en diferentes áreas de conocimiento. Las carreras profesionales de pregrado ofrecidas se concentran en las áreas de Salud, Ingeniería, Pedagogía y Ciencias Sociales y, en menor medida, en las áreas de administración y economía y ciencias. En cambio, en las carreras técnicas de nivel superior, el mayor número se encuentra en el área de Ingeniería y Administración, con una oferta menor en el área de Pedagogía y Salud.

La estructura de la matrícula del sistema regional de educación superior ha evolucionado de la siguiente forma en el periodo 2011 - 2015:

En la Fig. 1 se observa que el crecimiento de la matrícula de pregrado, a nivel regional, ha sido lento en los últimos cinco años, acumulando un aumento neto de 287 estudiantes en el periodo analizado. Cabe destacar que en el contexto global del sistema de educación superior chileno, la matrícula de la región de Magallanes al año 2015 representa un $0.67 \%$ del total nacional $(\mathrm{N}=1.165 .654)$ de acuerdo a las estadísticas del Servicio de Información de Educación Superior (MINEDUC-SIES, 2015). En este contexto, y considerando el foco del presente estudio, es interesante mostrar y analizar la distribución de la matrícula de pregrado según áreas de conocimiento y sexo a nivel nacional, lo cual permitirá contextualizar posteriormente la configuración disciplinaria de la propia Universidad de Magallanes, de su cuerpo docente y estudiantado. La distribución de la matrícula de pregrado a nivel nacional es la siguiente:

En la Tabla 1 se puede observar que, en términos generales, la distribución nacional de la matrícula de pregrado según sexo y área de conocimiento es relativamente homogénea y estable entre hombres y mujeres. Es decir, la mayoría de las áreas de conocimiento no tienen sesgos evidentes de género. Complementariamente, el crecimiento del sistema de educación superior chileno en los últimos cinco años se ha estabilizado, luego de dar un salto cuantitativo de la matrícula entre el año 2007 y 2010 cuando pasó de 750 mil a un millón de estudiantes. Después su crecimiento se tendió a ralentizar, prácticamente, en todas las áreas disciplinarias, salvo en el área de la tecnología que aumentó en 83 mil estudiantes, de los cuales mayoritariamente son hombres.

Por otra parte, y desde una perspectiva del desempeño de algunos indicadores específicos, la Universidad de Magallanes se caracteriza por poseer una población estudiantil que proviene mayoritariamente de la Región de Magallanes. Los establecimientos educacionales de origen de los estudiantes corresponden a un 31\% municipal, un $54 \%$ de los alumnos provienen de liceos particulares subvencionados y el $15 \%$ restante 
Tabla 1. Matrícula de pregrado nacional según área de conocimiento y sexo año 2011-2015.

\begin{tabular}{|c|c|c|c|c|c|c|}
\hline Area de conocimiento & Sexo & 2011 & 2012 & 2013 & 2014 & 2015 \\
\hline \multirow[t]{3}{*}{ Administracion y Comercio } & Hombres & 78.329 & 81.730 & 85.616 & 89.482 & 94.313 \\
\hline & Mujeres & 88.947 & 94.878 & 100.068 & 107.525 & 114.234 \\
\hline & Total & 167.276 & 176.608 & 185.684 & 197.007 & 208.547 \\
\hline \multirow[t]{3}{*}{ Agropecuaria } & Hombres & 14.730 & 14.195 & 14.110 & 13.094 & 12.906 \\
\hline & Mujeres & 13.309 & 12.912 & 12.746 & 12.927 & 13.081 \\
\hline & Total & 28.039 & 27.107 & 26.856 & 26.021 & 25.987 \\
\hline \multirow[t]{3}{*}{ Arte y Arquitectura } & Hombres & 27.430 & 26.610 & 26.049 & 25.145 & 24.692 \\
\hline & Mujeres & 27.231 & 27.361 & 27.406 & 27.377 & 27.614 \\
\hline & Total & 54.661 & 53.971 & 53.455 & 52.522 & 52.306 \\
\hline \multirow[t]{3}{*}{ Ciencias Basicas } & Hombres & 6.937 & 7.226 & 8.303 & 8.728 & 8.901 \\
\hline & Mujeres & 6.616 & 6.432 & 6.989 & 7.441 & 7.627 \\
\hline & Total & 13.553 & 13.658 & 15.292 & 16.169 & 16.528 \\
\hline \multirow[t]{3}{*}{ Ciencias Sociales } & Hombres & 32.291 & 32.991 & 33.401 & 33.995 & 34.939 \\
\hline & Mujeres & 69.554 & 72.742 & 75.766 & 78.442 & 80.463 \\
\hline & Total & 101.845 & 105.733 & 109.167 & 112.437 & 115.402 \\
\hline \multirow[t]{3}{*}{ Derecho } & Hombres & 21.034 & 20.181 & 19.981 & 19.503 & 19.075 \\
\hline & Mujeres & 22.777 & 22.088 & 21.324 & 21.106 & 21.251 \\
\hline & Total & 43.811 & 42.269 & 41.305 & 40.609 & 40.326 \\
\hline \multirow[t]{3}{*}{ Educacion } & Hombres & 43.584 & 43.245 & 41.451 & 38.750 & 36.933 \\
\hline & Mujeres & 89.555 & 90.909 & 88.149 & 86.642 & 84.040 \\
\hline & Total & 133.139 & 134.154 & 129.600 & 125.392 & 120.973 \\
\hline \multirow[t]{3}{*}{ Humanidades } & Hombres & 3.899 & 3.902 & 3.935 & 3.877 & 3.771 \\
\hline & Mujeres & 6.360 & 6.158 & 6.149 & 6.194 & 5.941 \\
\hline & Total & 10.259 & 10.060 & 10.084 & 10.071 & 9.712 \\
\hline \multirow[t]{3}{*}{ Salud } & Hombres & 50.404 & 53.633 & 55.202 & 54.011 & 53.940 \\
\hline & Mujeres & 142.490 & 155.975 & 160.283 & 165.308 & 169.116 \\
\hline & Total & 192.894 & 209.608 & 215.485 & 219.319 & 223.056 \\
\hline \multirow[t]{3}{*}{ Tecnología } & Hombres & 215.026 & 229.408 & 250.947 & 264.943 & 272.401 \\
\hline & Mujeres & 54.121 & 61.495 & 75.814 & 79.562 & 79.862 \\
\hline & Total & 269.147 & 290.903 & 326.761 & 344.505 & 352.263 \\
\hline \multirow[t]{3}{*}{ Sin área } & Hombres & 410 & 461 & 354 & 296 & 277 \\
\hline & Mujeres & 355 & 381 & 256 & 257 & 277 \\
\hline & Total & 765 & 842 & 610 & 553 & 554 \\
\hline \multirow[t]{3}{*}{ Total } & Hombres & 494.094 & 513.582 & 539.349 & 551.824 & 562.148 \\
\hline & Mujeres & 521.315 & 551.331 & 574.950 & 592.781 & 603.506 \\
\hline & Total & 1.015 .389 & 1.064 .913 & 1.114 .299 & 1.144 .605 & 1.165 .654 \\
\hline
\end{tabular}

Fuente: Estadísticas de la Educación (Ministerio de Educación de Chile, 2015, p. 110). 
proviene de liceos particulares pagados. Un $11 \%$ de los alumnos que ingresan vía PSU, lo hacen con un puntaje promedio (lenguaje-matemática) entre 475 y 500 puntos; un 64\% entre 501 y 600 puntos, un $19 \%$ entre 601 y 700 y un $0,9 \%$ superior a 700 . Por otra parte, la dotación de académicos a diciembre del año 2014, es de 204 profesionales (en régimen de planta y a contrata), de ellos un $60,8 \%$ tiene formación de postgrado, un $27,9 \%$ posee grado académico de doctor y un $32,8 \%$ grado de magíster. El personal académico alcanza a 374,04 jornadas completas equivalentes.

\section{METODOLOGÍA}

El programa global de investigación fue orientado a cartografiar diversos nodos críticos (estructurales, curriculares y epistemológicos) inherentes al desarrollo significativo, sustentable $y$ pertinente de esquema de trabajo intertransdisciplinario en el contexto universitario chileno. Los criterios de inclusión consideraron universidades chilenas con las siguientes características estructurales: instituciones tradicionales y de vocación pública, definidas en sus directrices políticas como universidades complejas (desarrollo de docencia, investigación y extensión), formación de pregrado y postgrado, acreditadas institucionalmente ante la CNA, y cuya organización incluyera al menos, la presencia de Facultades en el área de las Humanidades, Ciencias Básicas, Ciencias de la Salud y Ciencias de la Ingeniería. El presente artículo, reporta, profundiza y discute los resultados asociados a la investigación desplegada en la Región de Magallanes.

El estudio comprendió una observación de segundo orden orientada a la observación de observadores (Flores, 2009) y sus respectivas observaciones. Siguiendo a Arnold (2004), el núcleo de interés fue observar lo que los observados indican y describen, "qué observan", como también captar los esquemas diferenciales con que marcan tales observaciones y trazan distinciones, "cómo observan». Eneste sentido, las macro-orientacionesse centraron en el principio de sinergia, orientado hacia la identificación de redes o conjuntos relacionados de distinciones y no sólo a la reducción analítica y causal de componentes y procesos aislados. Es decir, redes de observaciones que se sostienen unas a otras en el contexto de los procesos de significación en torno a la noción de inter-transdisciplinariedad y sus proyecciones a los dominios de desarrollo de las universidades contemporáneas desde enfoques contextuales y situados.

\section{Recursos para la generación de información}

Las estrategias de generación de información contemplaron entrevistas en profundidad y grupos focales con estudiantes y académicos pertenecientes a diversas Facultades y carreras de pregrado. El desarrollo de entrevistas en profundidad (Guber, 2001) y grupos focales (Flores, 2009), quedó definido bajo el marco de eventos comunicativos controlados, pero no directivos, orientados a estimular los procesos de observación dentro de las cotas comunicativas del estudio. Los procesos de entrevista fueron, por tanto, flexibles y dinámicos; esto involucró una conversación abierta a la contingencia (Taylor \& Bogdan, 1996). Los grupos focales, dada su densidad para la construcción multicriterial y por sus potenciales participativos y de autoconocimiento grupal, posibilitaron convertir colectivos de discusión en dispositivos dinámicos de autorreflexión (Espina, 2007).

\section{Criterios de selección de informantes claves}

Dado el carácter predominantemente cualitativo, se consideró una selección estructural de informantes, relevando la representatividad de campos disciplinares y profesionales, así como criterios asociados a estamento (estudiantes y académicos) y trayectoria.

En total se realizaron siete entrevistas con académicos y dos con estudiantes, lo que posibilitó diagnosticar los principales elementos de contexto para el diseño de los protocolos de preguntas de los grupos focales posteriores. Para esta etapa preliminar de recolección de datos, se emplearon entrevistas de tipo semiestructurado, con el fin de entablar un diálogo gradual de participación en base a preguntas gran tour (Guber, 2001). Cada pregunta intentó abordar tópicos de coyuntura, sea ésta de tipo conceptual o de tipo metodológico. Posteriormente, fueron realizados seis grupos focales, dos con académicos y cuatro con 
Tabla 2. Distribución de agentes en cada grupo focal.

\begin{tabular}{|c|c|c|c|c|}
\hline $\begin{array}{l}\text { Participación de informantes } \\
\text { /Agenciamiento disciplinar }\end{array}$ & $\begin{array}{l}\text { Ciencias Sociales y } \\
\text { Humanidades }\end{array}$ & Ciencias Naturales & $\begin{array}{l}\text { Ciencias de la } \\
\text { Ingeniería }\end{array}$ & Ciencias de la Salud \\
\hline $\begin{array}{l}\text { Estudiantes participantes en } \\
\text { Grupos focales }\end{array}$ & 8 & 7 & 6 & 7 \\
\hline $\begin{array}{l}\text { Académicos participantes } \\
\text { Grupos focales }\end{array}$ & 5 & 4 & 2 & 2 \\
\hline Total & 13 & 11 & 8 & 9 \\
\hline
\end{tabular}

Fuente: elaboración de los autores.

estudiantes agenciados a los diversos sistemas disciplinares-profesionales considerados dentro de los límites de la investigación. La Tabla 2 muestra la distribución de la representatividad de agentes en los seis grupos focales realizados.

\section{Cotas comunicativas de la investigación}

Las cotas comunicativas quedaron definidas como los espacios de comunicación pertinentes al estudio, en este caso, todos aquellos tópicos que generaran significado para los informantes claves en el contexto de la prospectiva transdisciplinaria en la formación universitaria en Chile $y$, en particular, la Región de Magallanes. Esto implica, todos los procesos de significación que estuvieron a la base del amplio rango de interpretaciones. Para ello, se consideraron aquellos dominios cuya relación generó significado para los informantes claves en el contexto comunicativo abierto por las implicancias de la noción de intertransdisciplina en los esquemas de organización del conocimiento desplegados en los diversos dominios de desarrollo universitario en la región.

\section{Análisis e interpretación de información}

Las entrevistas y grupos focales fueron registrados mediante audio digital y transcritos integralmente, según el consentimiento informado por parte de los informantes, resguardando su identidad. Preliminarmente, se procedió a un proceso de codificación inicial que complementó estrategias de carácter inductivo y deductivo, a la vez de una triangulación intersubjetiva en base a tres codificaciones paralelas y simultáneas.

Para ello se generó un proceso de codificación a partir del marco teórico global que ofició de base a la investigación y, simultáneamente, desde el análisis inductivo de las principales regularidades cualitativas y levantamiento de categorías centrales, en correlato con los diversos significados expresados por los informantes en los grupos focales y entrevistas en profundidad realizadas. Considerando criterios de saturación de la información cualitativa levantada y la fusión de códigos basada en una lógica de síntesis y correspondencia, fue definido un total de 40 códigos distribuidos en 3 categorías: dominio conceptual global, dominio organizacional/ curricular, y dominio de desarrollo universitario.

El proceso de análisis posibilitó dos niveles de interpretación. En primera instancia, se analizó y comparó la distribución de frecuencias de códigos, con el objetivo de caracterizar los campos de significado en los cuales se movieron las distinciones generadas por los informantes claves. En un segundo momento, se modelaron y graficaron los efectos de asociación de los códigos desde una perspectiva sistémica y reticular. El criterio basal para la construcción de dendrogramas y redes de asociatividad, fue la coocurrencia de códigos dentro de un mismo campo semántico, definido este último como cada unidad de intervención de un informante en el contexto de una pregunta específica. El coeficiente de Jaccard (tolerancia 0,00001) fue el indicador específico de ocurrencia de segmentos superpuestos o adyacentes utilizados en la investigación. La complementariedad metodológica de los análisis de frecuencia e interacción de códigos observados en los eventos comunicativos, supone que no sólo es relevante la diversidad y frecuencia de codificación, sino también los efectos de interacción de estos elementos de sentido en redes de significación que se auto-eco-organizan. 


\section{RESULTADOS}

\section{Análisis de frecuencia de codificación}

En primera instancia, se levantó información referida a los indicadores de frecuencia de codificación globales asociados a los grupos focales realizados.

En relación a lo anterior, es posible observar que el código "inter-transdisciplina como principio de valor" es un código altamente recurrente, con un $13,6 \%$ de presencia en los diversos grupos focales. Esto reafirma que la noción de inter-transdisciplina es un elemento de sentido con amplio valor social y académico para los informantes claves participantes de la investigación. No obstante, esta amplia y distribuida valoración se expresa de formas muy diversas en términos de los elementos de sentidos adyacentes a ella, en correlato con los diversos campos disciplinares y el rol (académicos y estudiantes) de los informantes claves. Si bien códigos como "condicionante organizacional y comunicacional" (7,6\%), "desarrollo de docencia y formas de aprendizaje" (7,3\%), "formación y trabajo situado" (7,0\%), "baja interacción de carreras" $(6,0 \%)$ y "condicionante de temporalidad" (4,3\%), presentan una alta frecuencia global, su distribución específica en cada grupo focal varió significativamente. Esto da cuenta de la diversidad de contextos de significación en que la prospectiva transdisciplinar es inscrita, $e$ interpretada, por los diversos agentes y sus campos de referencia disciplinar. La siguiente Tabla (3) muestra las frecuencias y promedios globales por cada código, como asimismo, los efectos de distribución y dispersión de frecuencias de codificación para los seis grupos focales realizados. La categoría A corresponde al dominio conceptual global; la categoría $B$ al dominio de desarrollo universitario; $y$ la categoría $C$, al dominio organizacional y curricular.

\section{Análisis de efectos de relación de códigos}

En esta instancia fueron analizados los patrones de asociatividad de los códigos con mayor distribución de frecuencia: "inter-transdisciplina como principio de valor" (13,6\%), "condicionante organizacional y comunicacional" (7,6\%), "desarrollo de docencia y formas de aprendizaje"
(7,3\%), "formación y trabajo situado" (7,0\%), "baja interacción de carreras" $(6,0 \%)$ y "condicionante de temporalidad" (4,3\%). En el siguiente dendrograma (Fig. 2) se muestra los conglomerados en los que dichos códigos presentan efectos de mediación sistémica, en términos de los eventos de co-ocurrencia observados en los seis grupos focales.

En términos de aportar a la visualización de los efectos de interacción entre los códigos pertenecientes a los diversos dominios, la siguiente red global (Fig. 3) muestra las principales relaciones de códigos (en base a co-ocurrencia) intra e interdominios presentes en los seis grupos focales realizados. Para la lectura de la Fig. 3, se precisa que los efectos de interacción interna más intensa están representados por líneas más gruesas. De ahí que, en términos generales, el código "inter-transdisciplina como principio de valor" aparece, principalmente, asociado a los códigos "formación y trabajo situado y desarrollo de docencia y formas de aprendizaje", lo que evidencia su principal campo de significado. Otros códigos significativamente asociados aparecen a nivel de "baja interacción de carreras", "desarrollo de enfoques sistémicos y contextuales" y "aprendizaje desde la diversidad".

\section{DISCUSIÓN Y CONCLUSIONES}

La comprensión de la prospectiva intertransdisciplinaria en educación, frecuentemente, ha centrado su interés en aspectos estrictamente teóricos, en detrimento de estudios rigurosos e investigaciones de campo (Lenoir \& Sauvé, 1998). En ese sentido, el levantamiento y discusión de resultados espera contribuir a lograr un sustrato de interpretación de diversos fenómenos y problemáticas relacionadas a la organización del conocimiento y sus implicancias epistemológicas y políticas en la interfaz universidad $\leftarrow \rightarrow$ sociedad. Para Nicolescu (1996, 2008), el desarrollo progresivo de la perspectiva transdisciplinaria se ha acompañado del peligro de múltiples desviaciones: la desviación mercantil, la desviación de la búsqueda de nuevos modos de dominación, como asimismo la tautología y trivialización epistemológica. En consecuencia, asumiendo la relativa transversalidad de la noción de inter-transdisciplinariedad como principio de valor académico en el ámbito universitario local y global, se hace necesario 
Tabla 3. Distribución de frecuencias globales por códigos y categorías.

\begin{tabular}{|c|c|c|c|c|c|c|c|c|c|}
\hline Categorías & Códigos & ACCiencia & ACCs. Sociales & ES Ciencias & ES Cs. Sociales & ESCs. Salud & ES Cs. Ingeniería & Global & \% Global \\
\hline \multirow[t]{27}{*}{ A } & Abordar fenómenos globales y tronsdisciplincrios & & 2 & & & & & 2 & $0,70 \%$ \\
\hline & Aprendizaje desde la diversidad & & & & 4 & & & 4 & $1,30 \%$ \\
\hline & Centrolismo & & 1 & & 1 & & & 2 & $0,70 \%$ \\
\hline & Competencia e individualismo & & & 2 & 4 & 2 & & 8 & $2,70 \%$ \\
\hline & Condicionantes políticas & & & 1 & & & & 1 & $0,30 \%$ \\
\hline & Condicionantes sociales y económicas & & 1 & & & & & 1 & $0,30 \%$ \\
\hline & Desarrollo de enfoques sistémicos y contextuales & & & & 2 & & & 2 & $0,70 \%$ \\
\hline & Desvinculoción Ciencia y Hum anidades & & & & & & 2 & 2 & $0,70 \%$ \\
\hline & Estereotipos de identidad disciplinar & 1 & & & & 1 & & 2 & $0,70 \%$ \\
\hline & Formación y trabajo situado & & 1 & 4 & 5 & 10 & 1 & 21 & $7,00 \%$ \\
\hline & Implicancias éticas y valóricas & & 2 & & & & & 2 & $0,70 \%$ \\
\hline & Individialuzación y competencia & & & & & 1 & 4 & 5 & $1,70 \%$ \\
\hline & Inter-Transdisciplino como principio de velor & 12 & 3 & 3 & 9 & 13 & 1 & 41 & $13,60 \%$ \\
\hline & Lenguajes especializados y autorreferenciados & 1 & & & & & 1 & 2 & $0,70 \%$ \\
\hline & Necesidad de experticia disciplinar & & & 2 & & & & 2 & $0,70 \%$ \\
\hline & Necesidad especialización previo & & & & & 1 & 2 & 3 & $1,00 \%$ \\
\hline & Problemas motivacionales & & & & & & 1 & 1 & $0,30 \%$ \\
\hline & Problemótica de vinculación conocimiento general-profesional & 1 & 3 & 3 & & & 2 & 9 & $3,00 \%$ \\
\hline & Problemóticas asociadas al compromiso social del conocimiento & & & 3 & 6 & 2 & I & 15 & $5,00 \%$ \\
\hline & Problemóticas curricular y organizacional & 7 & 6 & & 1 & & | & 15 & $5,00 \%$ \\
\hline & Problemóticas de especialización & 1 & 2 & & & & & 3 & $1,00 \%$ \\
\hline & Problemóticas de madurez vocacional & & & & & 2 & & 2 & $0,70 \%$ \\
\hline & Problemáticas de validación y asimetría cientifica-profesional & 2 & 9 & & & 3 & & 15 & $5,00 \%$ \\
\hline & Problemáticas de vinculación nivel técnico y social-filosófico & 3 & 2 & & 3 & & 1 & 9 & $3,00 \%$ \\
\hline & Problemáticas socio-ambientales & & & & & & I & 3 & $1,00 \%$ \\
\hline & Superación del nivel de contenidos & 1 & & & 1 & & & 2 & $0,70 \%$ \\
\hline & Vinculación ortes-humanidades-ciencia & 6 & & & & & & 6 & $2,00 \%$ \\
\hline \multirow[t]{4}{*}{ B } & Desarrollo de Innovación y Tecnología & & & & & & I & 2 & $0,70 \%$ \\
\hline & Desarrollo de Investigación & 2 & 2 & 1 & & & & 5 & $1,70 \%$ \\
\hline & Desarrollo docencia y formas de aprendizaje & 9 & 3 & 3 & 3 & 3 & | & 22 & $7,30 \%$ \\
\hline & Desarrollo vinculoción con el medio & 1 & & 1 & & 1 & & 3 & $1,00 \%$ \\
\hline \multirow[t]{9}{*}{ C } & Baja interacción de carreros & & & 2 & 5 & 7 & | & 18 & $6,00 \%$ \\
\hline & Condicionante orgonizacional y comunicaccional & 2 & 2 & 6 & 4 & 9 & & 23 & $7,60 \%$ \\
\hline & Condicionante temporalidad & 2 & 1 & & 2 & 5 & I & 13 & $4,30 \%$ \\
\hline & Curriculum restringido y obligotorio & & & & 5 & 3 & | & 9 & $3,00 \%$ \\
\hline & Flexibilidad curricular restringida & & & 6 & 4 & 2 & & 12 & $4,00 \%$ \\
\hline & Instancias informales o iniciativas locales & 2 & 2 & 3 & 1 & 2 & & 10 & $3,30 \%$ \\
\hline & Obstáculos del Crecimiento organizacional & 1 & & & & & & 1 & $0,30 \%$ \\
\hline & Problemas de infroestructuro & & & 1 & & 1 & & 2 & $0,70 \%$ \\
\hline & Sobrecarga curricular y estres & & & & & 1 & & 1 & $0,30 \%$ \\
\hline
\end{tabular}

Fuente: elaboración de los autores.

abordar los complejos entramados de interpretación en torno a su proyección científica, política y social.

En este sentido, la prospectiva intertransdisciplinaria conlleva no sólo la transformación de aspectos estructurales en torno al sistema universitario, sino a la vez, el tránsito epistemológico a nuevos horizontes de sentido en torno a la organización de las formas de conocimiento y sus procesos de generación y comunicación. Ello involucra una interpretación abierta a los diversos rangos de sentido, desde donde operan los agentes educativos, en términos de cartografiar los sustratos 


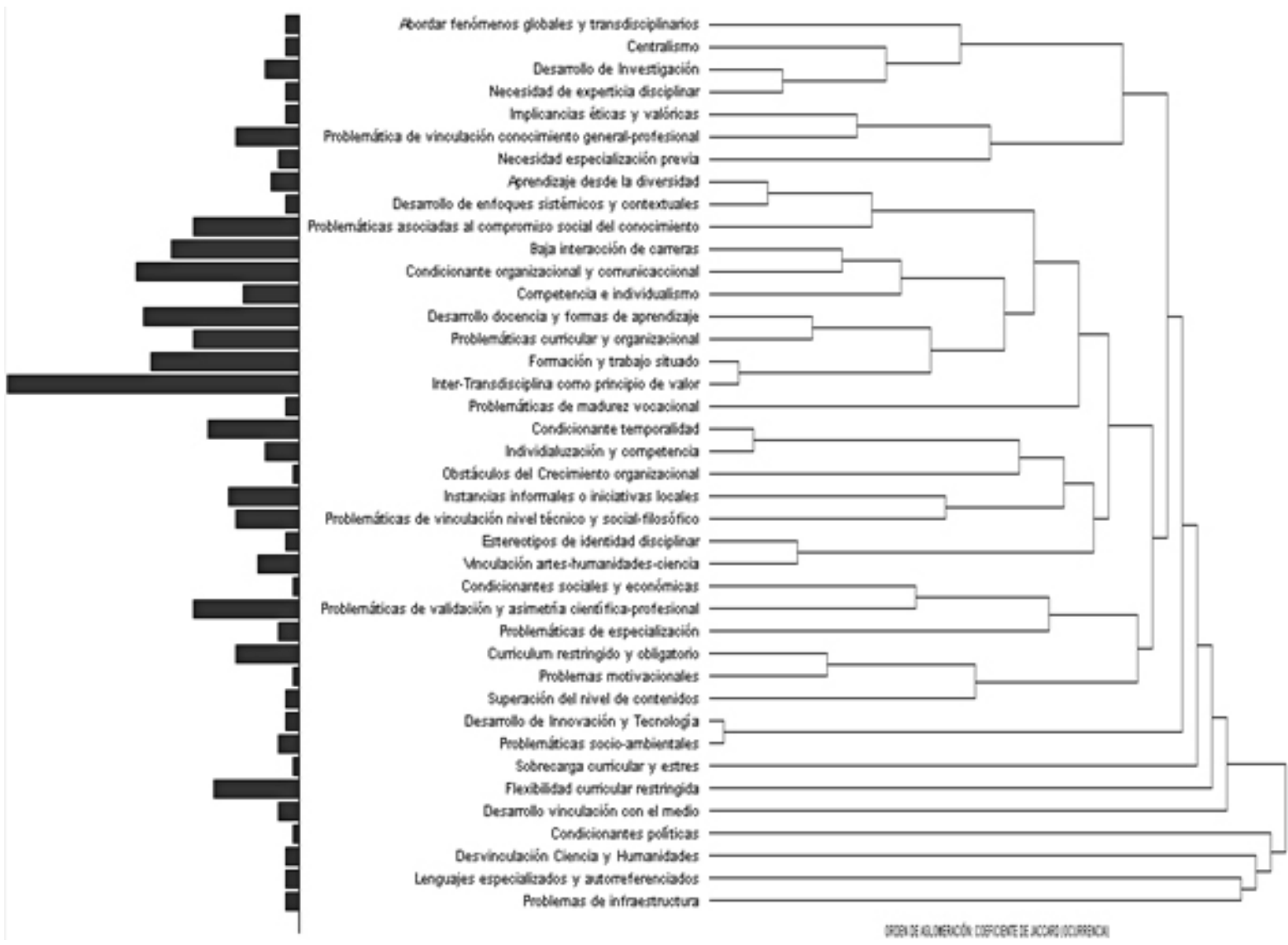

Fig. 2. Dendrograma asociado a los conglomerados de co-ocurrencia de códigos en los seis grupos focales.

epistemológicos que están a la base del desarrollo del sistema universitario en la región y el país.

En este marco de referencia, y desde el análisis de distribución de frecuencias de codificación, fue posible observar una amplia y distribuida valoración académica de la noción de inter-transdisciplinariedad en la educación superior en la región. Esta noción se expresó de distintas formas en términos de los elementos de sentidos adyacentes a ella, en correlato con los diversos campos disciplinares y el rol de los informantes claves. Lo anterior da cuenta de la diversidad de rangos de interpretación en que la prospectiva inter-transdisciplinar es significada por los diversos agentes y sus campos de referencia disciplinar, lo que simultáneamente se vincula con formas de interpretación transversales, como se discute más adelante.

Esta connotación heterogénea y extensa de la inter-transdisciplinariedad como principio de valor en el contexto universitario en la región, se manifestó con mayor significatividad en el campo de la formación y la docencia, tal como puede observarse en los análisis de distribución de codificación. Dentro de este contexto y en un rango de interpretación más específico, el análisis de asociatividad en torno a la prospectiva inter-transdisciplinaria muestra un significativo vínculo al ámbito de la práxis y la formación situada. Esta perspectiva fue levantada transversalmente por los diversos agentes, en especial estudiantes, los que movilizaron sus discursos intimamente situados a sus procesos de práctica profesional, instancias de profunda resignificación formativa y escenarios de alta pertinencia para el desarrollo de dinámicas inter-transdisciplinares, en términos profesionales y sociales. Este enfoque fue muy relevante en estudiantes agenciados a los campos disciplinares de Ciencias de la Salud y Ciencias Sociales y Humanidades.

Desde el protocolo metodológico desplegado con académicas(os), la noción de transdisciplina 


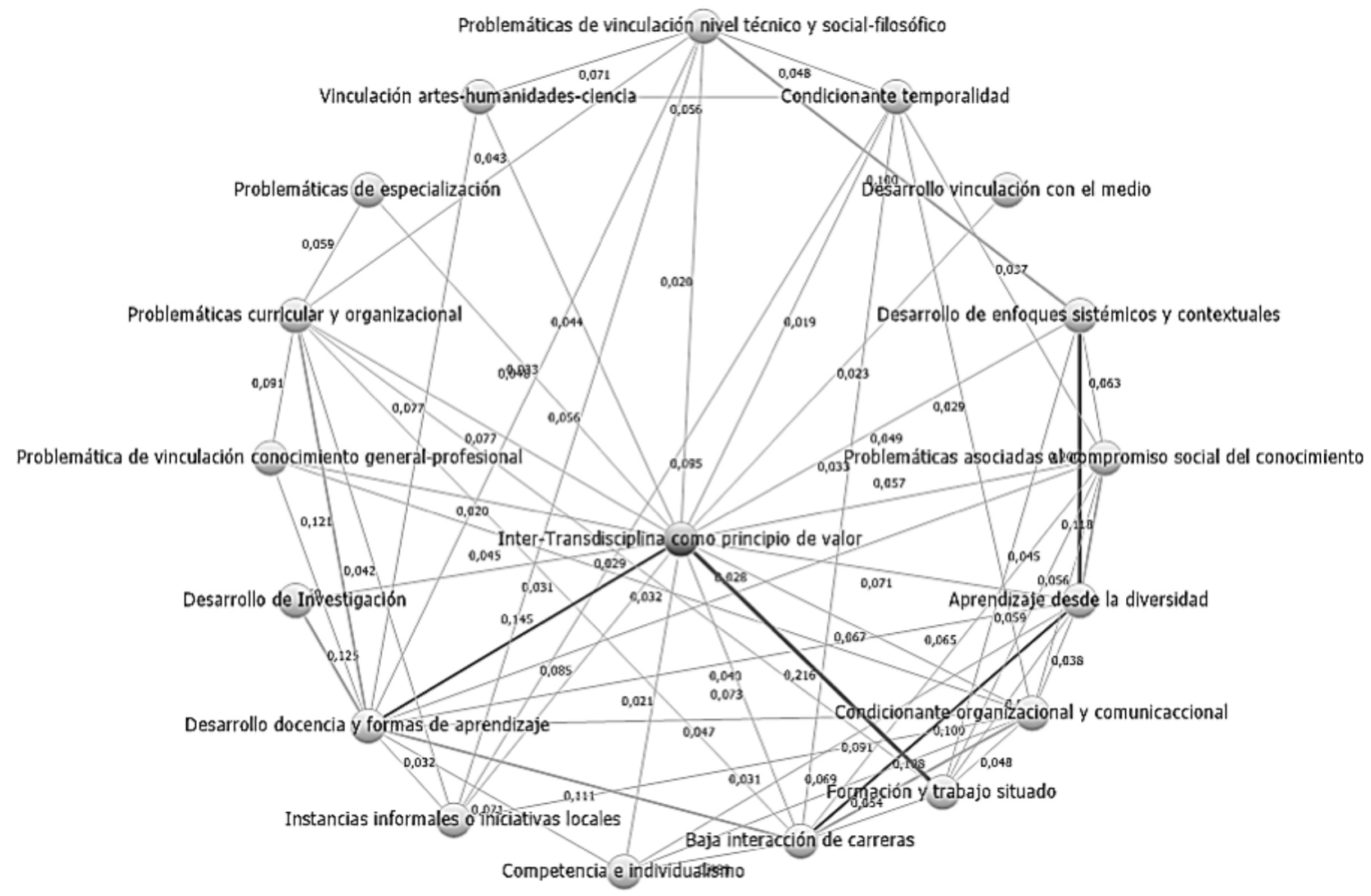

Fig. 3. Red global de las principales relaciones de códigos intra e interdominios presentes en los grupos focales.

es concebida transversalmente como un lenguaje que permite participar en torno a problemáticas comunes con diversas metodologías, relevando que la investigación no agota la posibilidad del diálogo disciplinar. A su vez, es considerada como una metodología que se presenta como necesidad y desafío, así como una posibilidad de transición e inflexión cualitativa en la organización del conocimiento. Se manifiesta que dejar la solución y abordaje de problemáticas en la interfaz naturaleza-cultura sea privativo de quienes estudian bajo estructuras de autorreferencia disciplinar, se transforma en un riesgo capital para el desarrollo social de la región. En términos de la mediación del código "vinculación con el medio", la contribución de un trabajo transdisciplinario estaría en función de acercar a la ciudadanía al empoderamiento de los temas que ocurren en la región, desde una investigación no elitista y compleja, que aporte al conocimiento regional desde un lenguaje alcanzable al común.

En convergencia con lo anterior, se enfatiza que el diálogo no debiese estar centrado en las disciplinas, sino en las personas; en otras palabras, es fundamentalmente un vínculo entre dimensiones vitales, que posibilite hacer praxis de vida, cultural, institucional, metodológica, como una dinámica de trabajo compleja en la que se construye una suerte de capacidad de vinculación cultural.

Además, desde la base del análisis de frecuencias de codificación, fue posible observar a nivel de las(os) estudiantes agenciados a diversos campos disciplinares, una perspectiva crítica a nivel curricular, expresada por la percepción de una baja interacción de carreras y flexibilidad curricular limitada, lo que es proyectado como una condicionante organizacional y curricular a la base de sus procesos formativos. De igual forma se reconoce como indispensable y necesario el diálogo de disciplinas desde la relación situada que se establece entre profesor(a)-estudiante.

A la vez, desde las(os) agentes académicas(os), se movilizó un discurso ampliamente distribuido en torno a la importancia de generar orgánicas de generación y comunicación de conocimiento más 
complejas e interactivas en términos disciplinares, las cuales son de fundamental importancia para la pertinencia social de la universidad en la región. Como condicionantes para el desarrollo de esta perspectiva, aparece la dimensión temporal y organizacional principalmente. Esto, debido a que diversos discursos aludieron al crecimiento de la organización universitaria como posible riesgo de aislamiento de los diversos dominios disciplinares en términos de investigación y docencia, al deprimirse gradualmente los espacios de conversación e iniciativa espontáneas. Complementariamente, se sostiene que la función interdisciplinaria estaría mediada por las cercanías de trabajo contextual bajo un programa de mayor generalidad, que por ejemplo pueden aportar las facultades en términos de integración. Sin embargo, esta mediación arriesga un tipo de relación funcional y teleológica inmediata que no alcanza dimensiones epistemológicas más profundas, por lo que sólo se alcanzaría un nivel de contacto multidisciplinar, más que inter-transdisciplinar.

Simultáneamente, en el estamento académico también aparecen códigos referidos a la problemática de validación y asimetría entre los diversos campos disciplinares, lo que no refleja un nodo crítico a nivel local, sino más bien a nivel de la comunidad científica nacional $e$ internacional y la centralidad de las instituciones públicas a su base. Se plantea una relativa dificultad de conmensurabilidad entre paradigmas tradicionales, por la brecha de una concepción de las ciencias exactas por sobre las humanidades y ciencias sociales. También se enfatiza, que el trabajo inter y transdisciplinario aumenta el valor y reconocimiento de las disciplinas (especialmente de las ciencias hacia las humanidades), por lo que este tipo de vinculación ofrece, además del diálogo, una especie de co-respeto disciplinar. En estos términos, la prospectiva inter-transdisciplinaria requiere una reflexión profunda en torno no sólo a la diversidad de sistemas de conocimiento, sino a la vez, en torno a los procesos epistemológicos de jerarquización y asimetría.

Complementariamente, el análisis de asociatividad de codificación muestra una alta mediación sistémica del código "intertransdisciplina como principio de valor", lo que más allá de su frecuencia de aparición, muestra una significativa relevancia en términos distribuidos para académicas(os) y estudiantes de los diversos campos disciplinares. De este modo es posible señalar que los códigos con mayor cercanía a esta noción, hacen referencia a la formación situada, formas de aprendizaje y problemáticas curriculares, lo que indica la importancia transversal de estos contextos de significación. Particularmente, en el estamento académico, se parte de una premisa común: la articulación entre los procesos de formación en la docencia universitaria, con lo cual se requiere que la formación general sea lo suficientemente unificadora y orientadora, de manera que cada una de las especialidades que cultiva la universidad tenga una articulación entre sí y una insoslayable pertinencia social.

Esto adquiere una importancia gravitante en un contexto social global, donde la prospectiva inter-transdisciplinaria parece proyectarse más significativamente, al menos en la política pública, en el ámbito del desarrollo de la investigación científica que en el ámbito curricular de la formación universitaria. No obstante, en el marco de este proyecto de investigación, ambas dimensiones conforman un bucle o dinámica recursiva epistemológica indisociable, en términos de la proyección pertinente y sostenible de la noción de inter-transdisciplina en la educación superior en la región y el país.

En síntesis, los resultados levantados permiten una cartografía preliminar en torno a los sustratos cualitativos donde se inscribe la prospectiva inter-transdisciplinaria en la educación superior en la región. La información cualitativa levantada, en triangulación con investigaciones previas, contribuye a la comprensión de nodos críticos y condiciones de emergencia relevantes (estructurales y no estructurales) para el despliegue de dinámicas inter-transdisciplinarias en la educación superior en la región y el país.

\section{BIBLIOGRAFÍA}

Arnold, M. (2004). Recursos para la investigación sistémico/ constructivista. En F. Osorio (Ed.), Ensayos sobre socioautopoiesis y epistemología constructivista. Santiago de Chile. Facultad de Ciencias Sociales. Universidad de Chile.

Capra, F. (1998). La trama de la vida. Barcelona: Anagrama. 
Capra, F. (2002). Las conexiones ocultas. Barcelona: Anagrama.

Cross, R., \& Parker, A. (2004). The Hidden Power of Social Networks Understanding How Work Really Gets Done in Organizations. Harvard: Harvard Business School Press.

González, E. (2011). La nueva juventud y el proceso de transición entre la educación media y superior. Experiencias universitarias. Santiago de Chile: Editores CINDA.

Díaz, N., Soler, A., \& Oliva, I. (2013). Enfoques pluri-intertransdisciplinarios en la prospectiva de desarrollo universitario: Un estudio de casos. Estudios pedagógicos (Valdivia), 39(2), 115-127.

Espina, M. (2007). Complejidad, transdisciplina y metodología de la investigación social. Utopía y praxis latinoamericana, 12, 29-43.

Flores, R. (2009). Observando observadores: una introducción a las técnicas cualitativas de investigación social. Santiago de Chile: Ediciones UC. Pontificia Universidad Católica de Chile.

Flores-Malagón A., \& Millán de Benavides (Eds.) (2002). Desafíos de la Transdisciplinariedad. Bogotá: Pensar/P. Universidad Javeriana.

Gaete, M., \& Morales, R. (2011). Articulación del sistema de educación superior en Chile: posibilidades, tensiones y desafíos. Revista Calidad de la Educación 35, 51-89.

Guber, R. (2001) La etnografía. Método, campo y reflexividad. Bogotá: Editorial Norma.

Greckhamer, T., Kro-Ljungberg, M., Cilesiz, S., \& Hayes, S. (2008). Demystifying Interdisciplinary Qualitative Research Qualitative Inquiry. 14(2), 307-331.

Koch, T., Oliva, I., Valencia, S., \& Salazar, J. (2015). Prospectiva Transdisciplinaria en Educación Superior: Construcción de Perfiles Vocacionales según Grados de Flexibilidad Disciplinar en Estudiantes de Último Año de Nivel Secundario. Formación Universitaria, 8(3), 65-78.

Lenoir, Y. (2013). Interdisciplinariedad en educación: una síntesis de sus especificidades y actualización. Revista Interdisciplina, 1(1), 51-86.

Lenoir, Y., \& Sauvé, L. (1998). Introduction. L'interdisciplinarité et la formation à l'enseignement primaire et secondaire: quelle interdisciplinarité pour quelle formation?. Revue des sciences de l'éducation, 24, 3-29.
Max-Neef, M. (2003). Fundamentos de la transdisciplinariedad. Maestros Gestores de Nuevos Caminos, 29.

Max-Neef, M. (2004) Foundations of transdisciplinarity. Ecological Economics, 53, 5-16.

Ministerio de Educación (2015). Estadísticas de educación 2015. Santiago de Chile: Centro de Estudios del Ministerio de Educación.

Morin, E. (1992). El método. Las ideas. Madrid: Editorial Cátedra.

Morin, E. (1998). El método: El conocimiento del conocimiento. Madrid: Editorial Cátedra.

Morin, E. (2006). El método. Ética. Madrid: Editorial Cátedra.

Motta, R. (2000). Complejidad, educación y transdisciplinariedad. Signos universitarios, 37, 69-92.

Motta, R. (2002). Complejidad, educación y transdisciplinariedad. POLIS, Revista Latinoamericana, 1(3), 1-21.

Nicolescu, B. (1996). La transdisciplinariedad. Manifiesto. Traduc. Núñez y Gérard. Paris: Ediciones Du Rocher.

Nicolescu, B. (2008). Transdisciplinarity: Theory and Practice. New Jersey: Hampton Press

OCDE (2009). Educación Superior en Chile. Santiago de Chile: MINEDUC.

Oliva, I. (2008). Conocimiento, universidad y complejidad: bosquejos epistémicos y metodológicos para una vinculación transdisciplinaria. Estudios Pedagógicos, 34(2), 227-243.

Oliva, I., Koch,. T., \& Quintero, J. (2014). Inter/ transdisciplinariedad $y$ universidad: Patrones de desplazamiento e interactividad disciplinar. Magis. Revista Internacional de Investigación en Educación. 6(13), 99-112.

Piaget, J. (1970). Problémes généraux de la recherche insterdisciplinare et mécanismes communs. En Tendances principales de la recherche dans les sciences sociales et humaines. Premiére Partie: Sciences sociales (pp. 629-685). Paris: Unesco.

Somerville, M., \& Rapport, D. (Eds.) (2000). Transdisciplinarity: reCreating Integrated Knowledge. Oxford: EOLSS.

Taylor, S., \& Bogdan, R. (1996). Introducción a los métodos cualitativos de investigación. Barcelona: Paidós.

von Bertalanffy, L. (1976). Teoría General de los Sistemas. Editorial Fondo de Cultura Económica. México.

von Foerster, H. (1996). Las semillas de la cibernética. Barcelona: Gedisa. 